\title{
Multidisciplinary motion economy in the cardiothoracic intensive care unit
}

John N. Melvan, MD, PhD, and Michael E. Halkos, MD, MSc

\footnotetext{
From the Division of Cardiothoracic Surgery, Department of Surgery, Emory University School of Medicine, Atlanta, Ga.

J.N.M. is an integrated cardiothoracic surgery resident at Emory University. He was awarded an American Association for Thoracic Surgery scholarship to attend the 11th Annual Foundation for the Advancement of Cardiothoracic Surgical Care, Cardiovascular-Thoracic Critical Care Conference in Washington, DC, in 2014. M.E.H. is program director of the Integrated Cardiothoracic Surgery Residency at Emory University, Atlanta, Ga. Disclosures: Authors have nothing to disclose with regard to commercial support.

Received for publication Feb 17, 2015; revisions received Sept 2, 2015; accepted for publication Sept 22, 2015; available ahead of print Oct 23, 2015.

Address for reprints: John N. Melvan, MD, PhD, Division of Cardiothoracic Surgery, Department of Surgery,

Emory Clinic Building A, Suite 2223, 1365 Clifton Rd NE, Atlanta, GA 30322 (E-mail: jmelvan@emory.edu) J Thorac Cardiovasc Surg 2016;151:319-20

$0022-5223 / \$ 36.00$

Copyright (C) 2016 by The American Association for Thoracic Surgery

http://dx.doi.org/10.1016/j.jtcvs.2015.09.089
}

"Big [changes] demand cooperation. Inspection, standardization, the right incentive, the proper teaching, the least fatigue, the greatest welfare. We shall submit our work to measurement and act on the results." In 1915, Frank Gilbreth $^{1}$ introduced the engineering concept of motion economy to health care, urging hospitals to maximize utility and efficiency. Today more than ever, we appreciate that perfect surgical outcomes are critically dependent on optimal perioperative care. Here we advocate that best economy of motion in the cardiothoracic intensive care unit (CTICU) is critically dependent on multidisciplinary teams functioning as a single unit to uphold the standards of care for a dynamically changing patient. To provide an understanding of the challenge from the perspective of a junior integrated cardiothoracic surgery resident, this article is written from the viewpoint of the junior author (J.N.M.).

As an integrated cardiothoracic surgery resident, my experience with major perioperative complications pales in comparison with that of my senior residents, attending surgeons, and many CTICU team members. My unique curriculum is composed of instruction not just by surgeons but also various CTICU specialists, including cardiologists, intensivists, perfusionists, and pulmonologists. Each individual is a specialist in his or her field-held to the appropriate specialty standards-and provides unique educational perspectives for cardiovascular care. Joined by nurses, pharmacists, nutritionists, and respiratory therapists rounding in the CTICU, it is easy to appreciate that patient information is not presented with a single voice but rather by a congress of individuals sharing ideas and observations. Consensus in management becomes the product of team debate, literature review, and anecdotal experience.

Despite my few years of training to date, the successes and failures of this team-based, CTICU care have become readily apparent. Success is the product of well-prepared

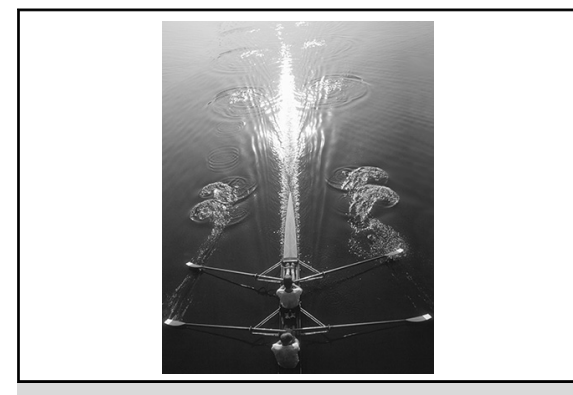

Teamwork and motion economy. (With permission http://www.andybaird.com/950/sculling.jpg).

\section{Central Message}

Multidisciplinary CTICU specialists are valuable consultants for surgeons who ultimately bear the responsibility for surgical patients.

See Editorial Commentary page 320.

team members, communicating effectively, and equally appreciating all concerns, so that a consensus can be reached to maximize patient benefit. ${ }^{2}$ Particularly for the CTICU patient, expedited identification, diagnosis, and modifications in treatment are critical for ensuring the best patient outcome. Failures occur easily. Missed details, poor communication, deviations from protocol, and informed neglect can produce devastating consequences. Few hospitalized patients have a more volatile course than an early postoperative cardiothoracic surgery patient. Failures as simple as dysfunctional equipment, lack of specialist availability, and poor postoperative sign-out can lead to postoperative complications, reoperations, and even death. Teaching CTICU motion economy remains challenging, but participation in multidisciplinary care teams and conferences by young trainees is an important start. All multidisciplinary team members should focus on providing optimal patient care in a collegial, team-oriented manner.

During cardiothoracic surgery residency, we are trained in dogma that has been developed during the last 100 years, mastering algorithms that have been rehearsed and perfected. The education of today's cardiothoracic surgery resident, however, is constantly evolving. The explosion of new surgical technologies provides incentive for creatively addressing old questions. Cutting-edge, unorthodox approaches, such as percutaneous valve replacement, robotic surgery, and mechanical assist devices, have prompted the development of new protocols and surgical 
approaches. Current CTICU leadership positions are composed of surgeons, anesthesiologists, cardiologists, and intensivists, each of whom specializes in specific elements of CTICU care. Cardiothoracic surgeons acknowledge that the current escalation in surgical technologies complicates an individual surgeon's ability to master all modalities of perioperative care. CTICU specialists are consulted to provide a synthesized understanding of these nonsurgical elements and to assist surgeons with decision making. Surgeons possess the greatest insight into patient-specific disease processes and procedure-specific technical details, however, and are best prepared to anticipate postoperative complications relevant to surgery. Moreover, current public reporting algorithms place sole responsibility for patient outcomes on individual surgeons. For these reasons, cardiothoracic surgeons remain ultimately responsible for the care of their patients and must continue to be the team leaders for postoperative care in the CTICU.

\section{References}

1. Gilbreth FB. Hospital efficiency from the standpoint of the efficiency expert. Boston Med Surg J. 1915;172:774-5.

2. Reader TW, Flin R, Mearns K, Cuthbertson BH. Developing a team performance framework for the intensive care unit. Crit Care Med. 2009;37:1787-93.

\title{
EDITORIAL COMMENTARY
}

\section{The devil is in the details}

\author{
Bryan A. Whitson, $\mathrm{MD}, \mathrm{PhD}$ \\ From the Division of Cardiac Surgery, Department of Surgery, Ohio State University Medical Center, Columbus, \\ Ohio. \\ Disclosures: Author has nothing to disclose with regard to commercial support. \\ Received for publication Oct 11, 2015; accepted for publication Oct 18, 2015; available ahead of print Nov 21, \\ 2015 . \\ Address for reprints: Bryan A. Whitson, MD, PhD, Director, End-Stage Cardiopulmonary Failure \\ Program, Co-Director, COPPER Laboratory, Division of Cardiac Surgery, Department of Surgery, Ohio State \\ University Medical Center, N-816 Doan Hall, 410 W 10th Ave, Columbus, OH 43210 (E-mail: bryan.whitson@ \\ osumc.edu) \\ J Thorac Cardiovasc Surg 2016;151:320-1 \\ $0022-5223 / \$ 36.00$

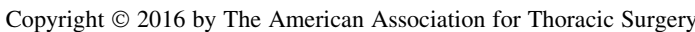 \\ http://dx.doi.org/10.1016/j.jtcvs.2015.10.045
}

\begin{abstract}
The patient's outcome correlates directly with the surgeon's attention to a myriad of minor details. This obsession of doing a lot of little things right is the foundation for good surgical results.
\end{abstract}

$$
\text { -Hiram C. Polk, MD }
$$

In this issue of the Journal, Melvan and Halkos ${ }^{1}$ provide us with a wonderful editorial on the paramount impact of the cardiothoracic intensive unit's care on our patient's postoperative course from a trainee's perspective. In reading the insightful article, 3 salient points come to the forefront: (1) the complex care in our cardiothoracic intensive care units is being performed by dedicated team members from multiple specialties; (2) as our advanced therapies open the door to the care of more ill patients, the importance of excellent, vigilant perioperative care cannot be understated; and (3) the cardiothoracic surgeon is the "captain of the ship."

The 21 st century cardiothoracic surgery intensive care unit (CTICU) is a complex creature. As cardiothoracic

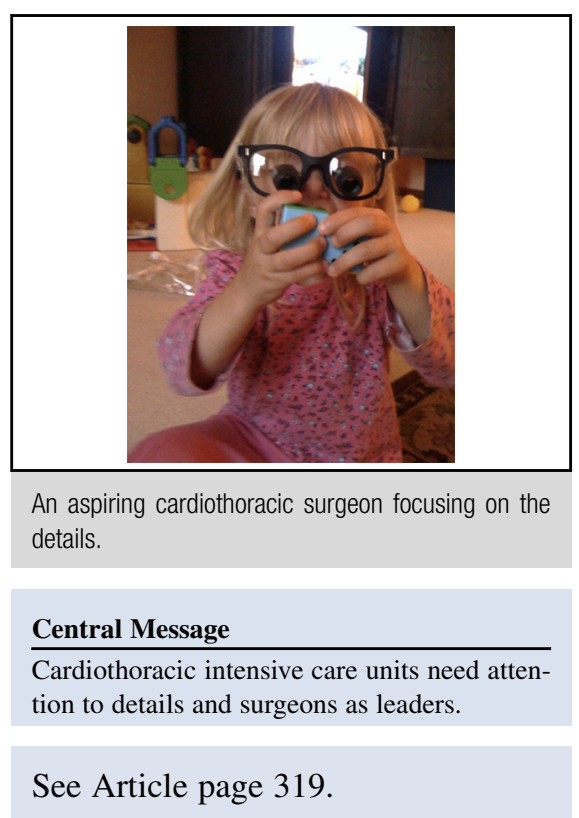

surgeons, we are extending the care that we provide to those more ill and with more nuanced needs than we could have ever imagined. As such, our CTICU team is equally complex and specialized-we draw on the strengths of our surgical and anesthesia critical care, pulmonology, cardiology, nephrology, pharmacy, palliative medicine, social work, and physical and occupational care colleagues. This care model does provide comprehensive care to these sick patients. ${ }^{2}$ Although we do have the benefit of these perspectives, however, there is a cautionary tale that we 